\title{
Study of influence of densification on control of conductivity and spectral characteristics of thin films of carbon nanotubes in terahertz frequency range
}

\author{
P. Demchenko ${ }^{1}$, D. Gomon ${ }^{1}$, I. Anoshkin ${ }^{1,2}$, S. Smirnov ${ }^{2}$, \\ D. Lioubtchenko ${ }^{1,2}$, M. Khodzitsky ${ }^{1}$ \\ ${ }^{1}$ Department of Photonics and Optical Information Technologies, ITMO University, Saint Petersburg, Russian, \\ pert.s.demchenko@gmail.com \\ ${ }^{2}$ Department of Micro and Nanosystems, KTH Royal Institute of Technology, Stockholm, Sweden.
}

The terahertz $(\mathrm{THz})$ frequency range has a large number of potential applications. Terahertz radiation interacts with rotational and vibrational transitions of organic molecules, thus, this one can be used for spectroscopy of small organic molecules, for example glucose [1], water [2], macromolecules, cells [3], biological _tissues and medicines is possible. Also, the terahertz frequency band can be used in quality control of electrical circuits, drugs and product, astronomy, security systems and communications [4].

Each application requires components for generation, detection, polarization, amplification, and modulation of terahertz radiation. Currently, devices are being developed, the parameters and properties of which can be controlled. Several materials are capable of changing the properties and characteristics in the terahertz frequency range one of them is single-walled carbon nanotubes (SWCNTs). So, in our last article [5] we showed that the complex dielectric permittivity of a thin film of carbon nanotubes under the influence of optical pumping varies in the terahertz frequency range. Such thin layers of nanostructures readily absorb various liquids. Therefore, such samples can be processed in various ways. One of them is densification. This is the process of compacting the material. In this paper, we investigated the optical properties of thin SWCNT layers before and after densification, and how the optical properties change under the influence of infrared pumping at wavelength of $980 \mathrm{~nm}$.

SWCNT film was made by chemical vapor deposition (CVD) [6] on nitrocellulose filter. This film was examined with scanning electron microscope (SEM) (Fig. 1(a)) and transmission electron microscope (TEM) to determine dispersion of length of nanotubes, their diameter and film's thickness.

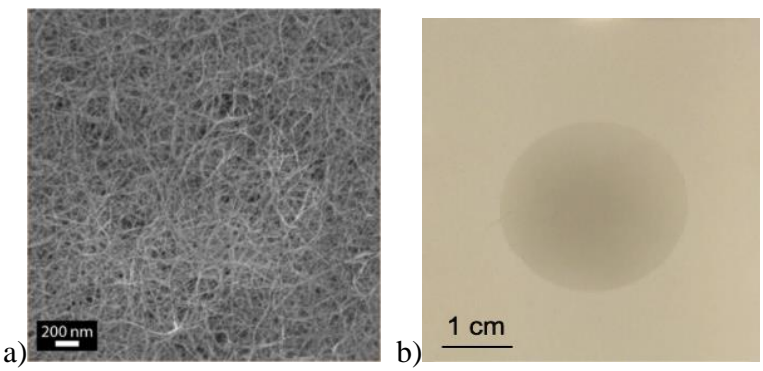

Fig. 1. a) Sample image of a SWCNT film obtained using a scanning electron microscope, b) Photo of sample
Characteristics of SWCNT film:

\begin{tabular}{|l|l|}
\hline Nanotubes's length & $\sim 15 \mathrm{um}$ \\
\hline Diameter of nanotubes & $1.3-2 \mathrm{~nm}$ \\
\hline Film's thickness & $55 \mathrm{~nm}$ \\
\hline
\end{tabular}

After that, SWCNT film was moved on teflon (PTFE) substrate with thickness of 350 um (Fig. 1(b)). The sample was studied using terahertz timedomain spectrometer (THz-TDS) [7] with additional $980 \mathrm{~nm}$ infrared laser for optical pumping (up to 0.7 $\mathrm{W} / \mathrm{cm}^{2}$ ). Scheme of THz-TDS is shown in Fig. 2.

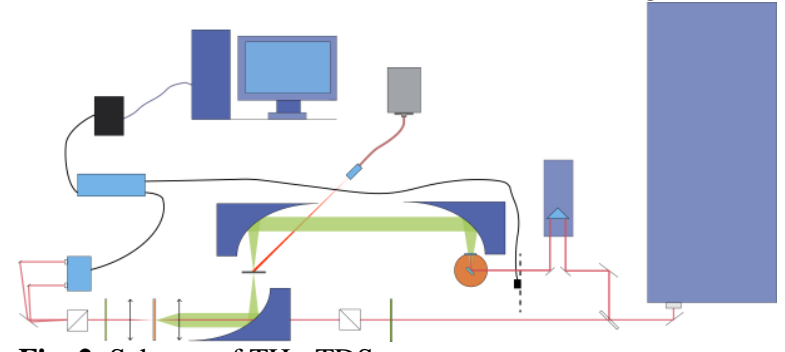

Fig. 2. Scheme of THz-TDS

$\mathrm{THz}$ waveforms were obtained before densification and after, with and without optical pumping (Fig. 3).

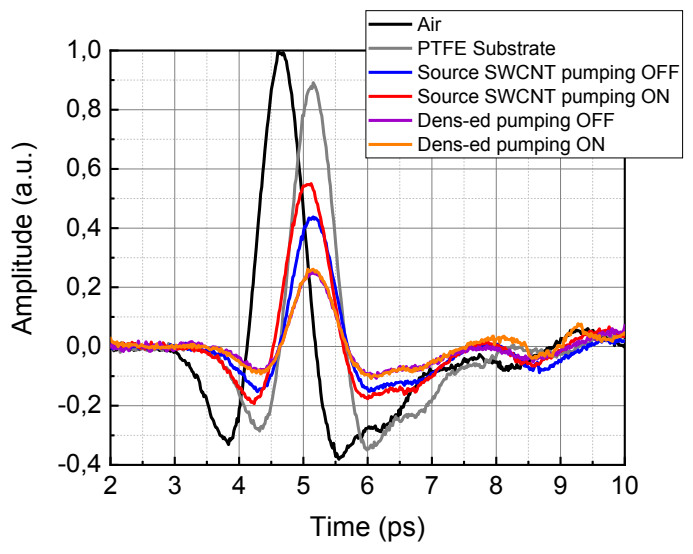

Fig. 3. Typical $\mathrm{THz}$ waveforms

To calculate a complex conductivity of the sample, the thin-film method [8] was used

$$
\hat{\sigma}(f)=\frac{1}{Z_{0}}\left(\hat{n}_{\text {Sub }}(f)+1\right)\left(\frac{\hat{E}_{0}(f)}{\hat{E}(f)}-1\right),
$$

were $Z_{0}$ - impedance of free space, $f$ - frequency, $n_{\text {Sub }}$ - refractive index of substrate, $\hat{E}_{0}(f)$ and $\hat{E}(f)$ - the Fourier transforms of the detected $\mathrm{THz}$ wave 
transmitted through the SWCNT layer on the PTFE substrate, and the PTFE alone, respectively.

Spectra of complex conductivity calculated by the formula (1) are shown in Figure 4.

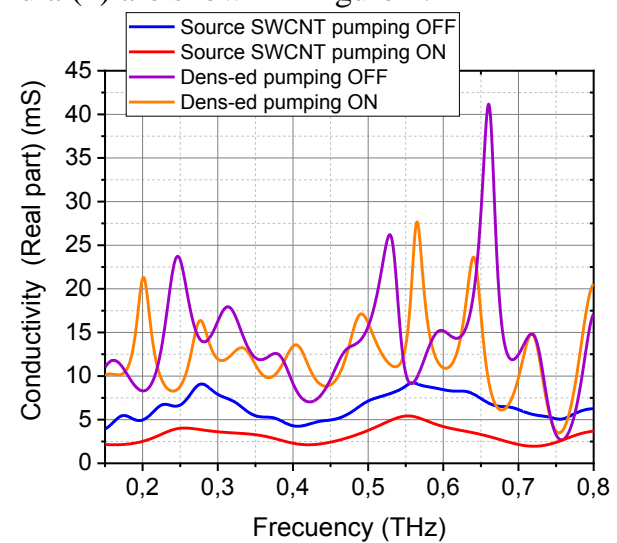

a)

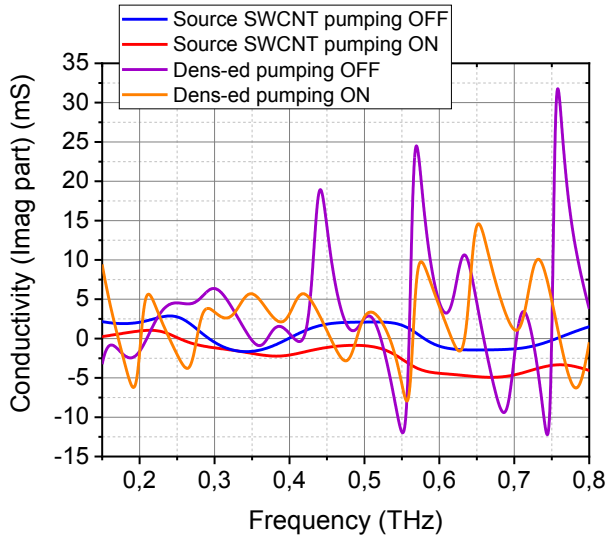

Fig. 4. Real part (a) and Imaginary part (b) of complex conductivity of thin-film SWCNT

The sample before densification has the effect of changing the complex conductivity (as in article 4). One explanation for this effect is that the nanotubes in the original sample are relatively free of each other, like "wool." Upon irradiation of a sample, the SWCNT oscillates more strongly; therefore, the contact area between adjacent nanotubes decreases and the conductivity of the sample decreases. During the process of densification the distance between the nanotubes decreases, the overall density of the film increases, hence the contact area between the tubes increases and the conductivity increases. This is confirmed in our work. After densification, the conductivity increased and does not change upon optical pumping. In addition, peaks appeared on the spectra after sample processing, presumably due to the uneven compaction of a thin-film and a decrease in the signal-to-noise ratio for densified sample.

According to the result of the work, it was obtained that after densification, the sample began to pass less (increased losses / increased the real part of the conductivity), the real part of the refractive index slightly increased. At the same time, the ability to control explicitly is almost imperceptible. This can be used in terahertz antennas and integrated circuits for the terahertz frequency range, where part of the nanotubes are densified to attenuate control by optical pumping.

\section{References}

1. Gusev S., Demchenko P., Cherkasova O., Fedorov $V$., Khodzitsky M. Influence of Glucose Concentration on Blood Optical Properties in THz Frequency Range // Chinese Optics 2018. V 11. No. 2. P. 182-189.

2. Borovkova M., Khodzitsky M., Demchenko P., Cherkasova O., Popov A., Meglinski I. Terahertz time-domain spectroscopy for non-invasive assessment of water content in biological samples // Biomedical Optics Express 2018. V. 9. No.5. P. 2266.

3. Borovkova M., Serebriakova M., Fedorov V., Sedykh E., Vaks V., Lichutin A., Salnikova A., Khodzitsky M, Investigation of terahertz radiation influence on rat glial cells // Biomed. Opt. Express, BOE 2017. V. 8. No. 1. P. 273-280.

4. Federici J., Moeller L. Review of terahertz and subterahertz wireless communications // Journal of Applied Physics 2010. V. 107. No. 11. P. 111101.

5. Smirnov S., Anoshkin I., Demchenko P., Gomon D., Lioubtchenko D., Khodzitsky M., Oberhammer J. Optically controlled dielectric properties of single-walled carbon nanotubes for terahertz wave applications// Nanoscale 2018. V. 10. No.26. P. 12291-12296.

5. Anoshkin I., Nasibulin A., Tian Y., Liu B., Jiang H., Kauppinen E. Hybrid carbon source for single-walled carbon nanotube synthesis by aerosol CVD method // Carbon 2014. V. 78. P. 130-136.

6. Bespalov, V., Gorodetskiľ, A., Denisyuk, I., Kozlov, S., Krylov, V., Lukomskiü, G., Putilin, S. Methods of generating superbroadband terahertz pulses with femtosecond lasers //Journal of Optical Technology. 2008. V. 75. No. 10. P. 636-642.

7. Tinkham M. Energy Gap Interpretation of Experiments on Infrared Transmission through Superconducting Films // Physical Review 1956. V. 104. No. 3. P. 845-846 